\title{
Fluidic Oscillators Mediating Generation of Microbubbles (Survey)
}

\author{
Václav Tesař
}

check for updates

Citation: Tesař, V. Fluidic Oscillators Mediating Generation of Microbubbles (Survey). Fluids 2021, 6, 77. https:// doi.org/10.3390/fluids6020077

Academic Editors: Rene Woszidlo and Oliver Krüger

Received: 17 December 2020

Accepted: 4 February 2021

Published: 9 February 2021

Publisher's Note: MDPI stays neutra with regard to jurisdictional claims in published maps and institutional affiliations.

Copyright: (C) 2021 by the author. Licensee MDPI, Basel, Switzerland. This article is an open access article distributed under the terms and conditions of the Creative Commons Attribution (CC BY) license (https:/ / creativecommons.org/licenses/by/ $4.0 /)$.
Institute of Thermomechanics, Czech Academy of Sciences, 18200 Prague, Czech Republic; tesar@it.cas.cz

\begin{abstract}
If a gas volume is distributed into many microbubbles of a sub-millimetre size, the total gas/liquid surface becomes very large. This increases overall heat and/or mass transport across the sum of surfaces. The paper discusses several applications in which the use of microbubbles increases efficiency of various processes, especially in wastewater treatment and in growing microorganisms such as algae, yeast, bacteria, or primitive fungi. The problem of microbubble generation by percolation in aerator is their coalescence into larger bubbles, whatever small are the pores in the aerator in which the microbubbles are generated. The solution of this size discrepancy question was found in agitating the gas flow by a fluidic oscillator prior to its injection through the aerator. The oscillator is a no-moving-part device, simple, inexpensive, resistant to external effects like acceleration or heat, and with long maintenance-free working life.
\end{abstract}

Keywords: fluidics; fluidic oscillator; fluidic amplifier; feedback loop; aerator; diffuser; biotechnology; microorganisms; wastewater treatment

\section{Introduction}

Microbubbles are small gas bubbles in a liquid, less than $1 \mathrm{~mm}$ in diameter. They have several useful properties, some that have already been recognised much earlier but have not been used in practical applications until about a decade ago. The reason was the energetically inefficient methods by which the microbubbles were generated. These early generation methods produced microbubbles that were rather unstable. A remedy for this instability was sought in addition of surfactants to the liquid. This, however, complicated both investigations and uses, especially due to the toxicity of some surfactants [1] and their generally adverse effects on living organisms. This has prevented the use of microbubbles in the otherwise promising area of biotechnology.

A change in this situation took place with the appearance of the patent [2]. It introduced the novel method of microbubble generation, based on gas agitation by a nomoving-part fluidic oscillator prior to the injection of gas into the liquid through pores of an aerator-a body with many small passages. The new low energy approach requires no surfactant and also minimises the lysis of organism cells by the bursting that takes place when, in their motion upwards, they reach the top surface of the liquid.

This novel approach to generation makes microbubbles an interesting and useful subject, bringing advantages applicable in many areas, in particular for reactors in biotechnology. The recent research activities and their result appear now in the literature at a high frequency. There are already even some early industrial activities. The publications feature in a wide range of various areas of technology as well as biology, so that it is easy to lose sight. The present paper is a survey intended to provide a basic orientation on what has been done. It is centred mainly on the activities taking part at the University of Sheffield in the U.K., where the idea originated.

\section{Size Discrepancy of Bubble and Aerator Pore}

The advantages obtained by the division of input gas volume into the large number of very small bubbles are based on the resultant large area of the total phase boundary 
between the gas and liquid phases. Since the intensity of transport processes (such as heat and/or mass transfer) across this phase boundary is proportional to the total surface area, it always seemed a quite obvious idea to increase the transfer intensity by making the bubbles very small. The simplest approach seemed to use for their generation an aerator with a large number of small parallel pores through which the gas is percolated. Typical present day commercially available aerators are mostly made by sintering a large number of very small objects (e.g., small spheres of thermos plastic polymer or glass spheres). Serving as the pores are then the small empty spaces left between these objects in the sintering process. Unfortunately, the experience with a usual aerator with steady gas flow percolation is almost universally disappointing. Irrespective of how small are the pore cross-sections, the generated bubbles are practically always substantially larger.

This discrepancy between the size of the pores and the size of generated bubbles was only relatively recently explained in [3]. The explanation was found in the conjunctions of the bubbles, schematically presented in Figure 1. An important role is the small bubble rise velocity law, presented in Figure 2, evaluated as a power-law fit through the data points for microbubbles from several experiments [4-8]. The equation of this law is:

$$
w=0.25 d^{2} / 3600
$$

where $w(\mathrm{~m} / \mathrm{h})$ is the terminal velocity of the upwards motion and $d(\mathrm{~mm})$ is the microbubble diameter. This Equation (1) assumes steady flow conditions in which the velocity is governed by equivalence of two acting forces, the hydraulic friction force on the microbubble boundary and of the driving force generated by the difference of specific volumes of the gas and liquid.

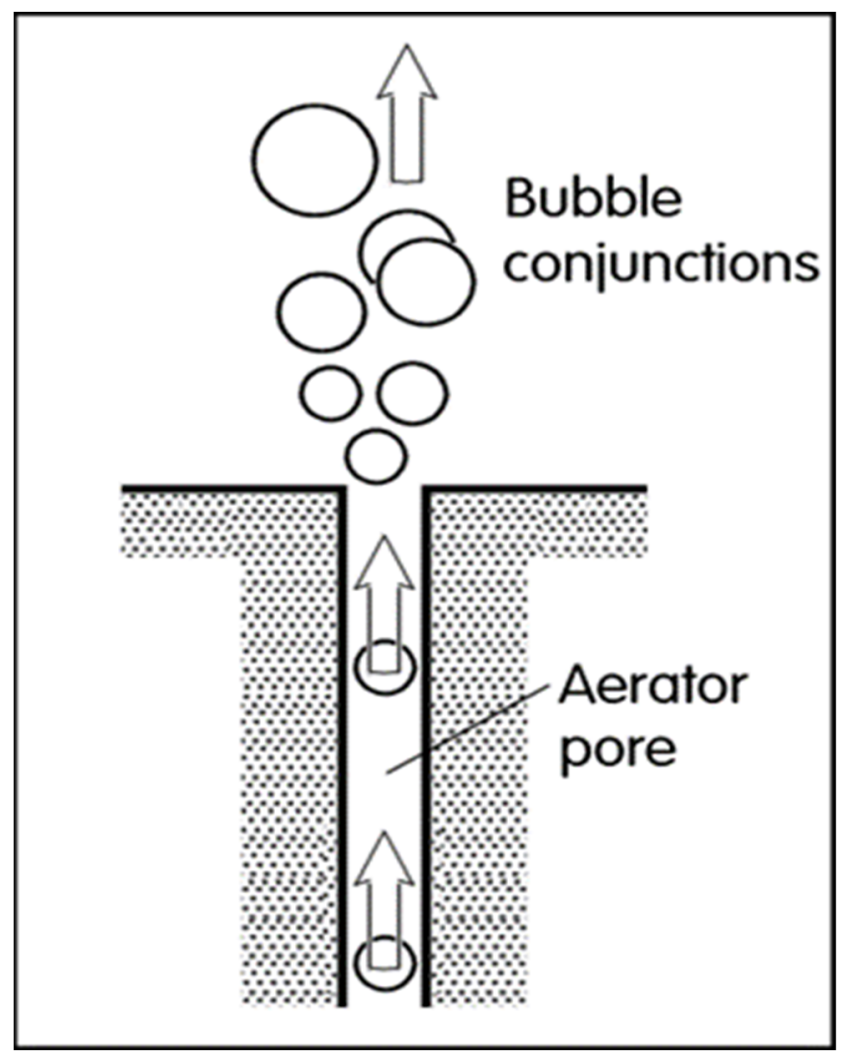

Figure 1. Representation of the conditions near the aerator pore exit with steady gas flow. The slowly moving microbubbles at the exit form larger bubbles by mutual conjunctions. 


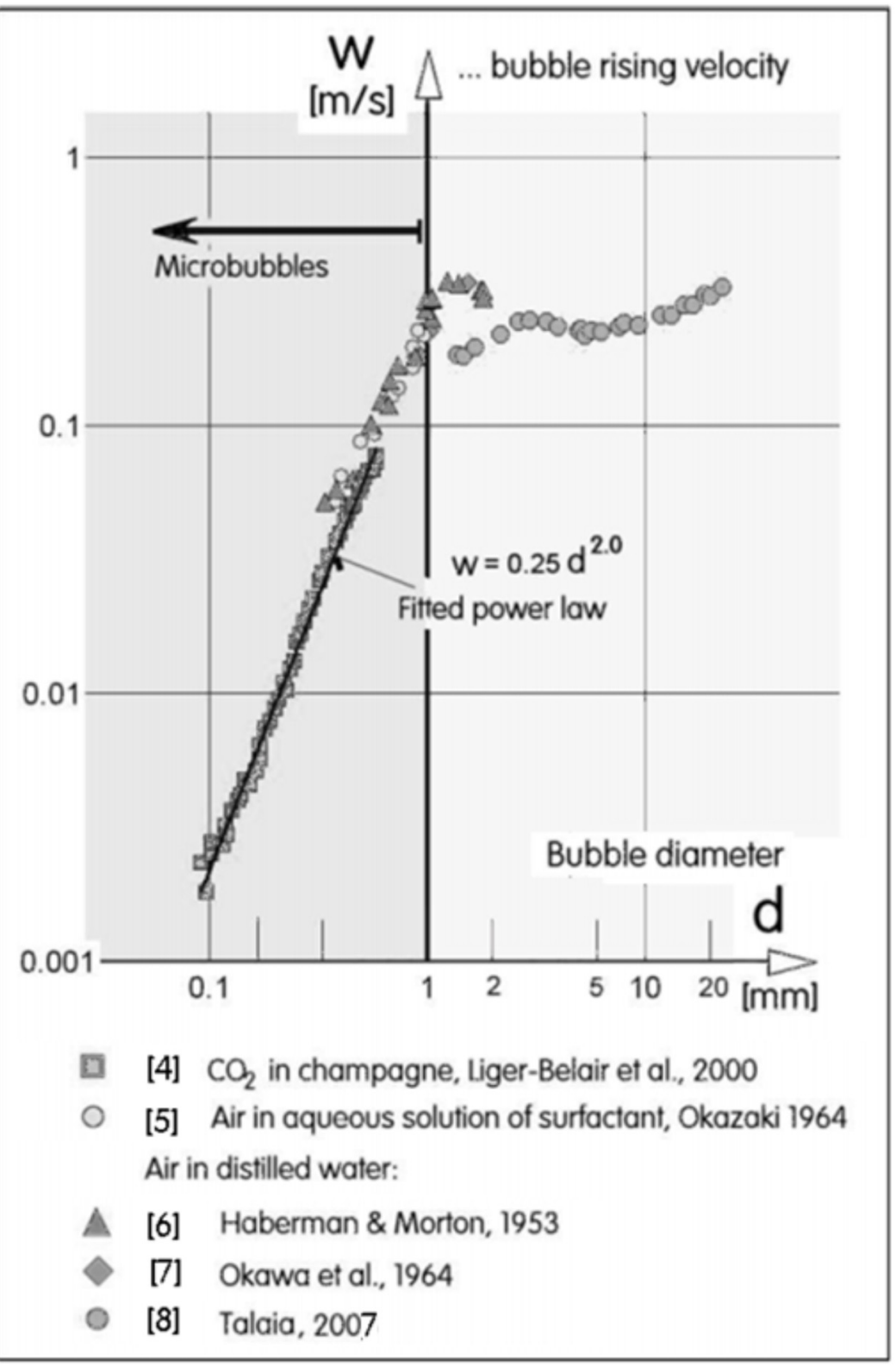

Figure 2. Terminal velocity of small bubbles rising in stationary liquid, from [9]. The velocity of microbubbles $(d<1 \mathrm{~mm})$ decreases rapidly with their decreasing diameter.

Evidently, the small microbubbles move very slowly. It may be noted in Table 1 that the $50 \mu \mathrm{m}$ microbubble (certainly not the smallest) moves so slowly that for traversing the whole height of a typical bioreactor vessel it needs the time scale of an hour. These long durations, of course, can have consequences such as the full finishing of transfer processes, for which there is not enough time during the fast moving rise of larger bubbles. In the situation presented in Figure 1, the onset of very low velocity of the microbubble after leaving the aerator pore exit, forms a bunch of very small bubbles near one another. As presented in the drawing, inside the aerator pore the microbubbles are initially fast moving and thus their size corresponds to the size of the pore cross section area. They move inside the pore at a relatively high velocity dictated by properties of the gas supply source. This velocity is generally much higher than the value in Equation (1). On leaving the pore exit, the bubble velocity suddenly decreases to the value smaller than the value shown in Figure 2, which means a near halt of motion. The microbubble only slowly increases its upwards motion velocity to the fully developed terminal velocity given by Equation (1).

The nonlinearity of this velocity law is seen from several evaluated data points: 
Table 1. Terminal velocity of microbubbles rising in liquid.

\begin{tabular}{cc}
\hline Diameter & Rising Velocity \\
\hline $0.1 \mathrm{~mm}$ & $9 \mathrm{~m} / \mathrm{h}$ \\
\hline $0.05 \mathrm{~mm}$ & $2.25 \mathrm{~m} / \mathrm{h}$ \\
\hline $0.02 \mathrm{~mm}$ & $0.36 \mathrm{~m} / \mathrm{h}$ \\
\hline
\end{tabular}

This sudden velocity decrease and very slow motion at the pore exit means the newly emerging microbubble on leaving the pore inevitably collides with its predecessor microbubble, which is still dwelling near the pore exit. The collision and subsequent conjunction into a single larger bubble is more inevitable because the formation of a larger bubble is associated with decrease of total energy of surface tension (note that the total surface energy is smaller in the single larger bubble than in the sum of the two original microbubbles of the same total volume) and all passive processes always move into the direction of energy decreasing.

The energy released in the conjunction is used for oscillating the resultant larger bubble after the conjunction is over. This way, the energy is dissipated. Bubble oscillation increases its hydrodynamic resistance, which is an additional effect that decreases and almost stops the rising motion [10]. The microbubble thus stays at or near the pore exit for some time. This results in conjunction with yet another of the later coming follower microbubbles. Thus at the pore exit dwells a bunch of bubbles, each of them stepwise increasing in diameter with each performed collision. The repeated processes finally end with a large bubble, substantially larger than the size of an aerator pore exit and capable of rising sufficiently fast away from the aerator pore.

The reason for the capability to keep the size of generated bubbles small by gas flow oscillation at the aerator was discovered from high-speed camera images. The original idea of why the oscillation is useful was the expectation of it breaking the larger bubbles into smaller ones by oscillatory decomposing. Later detailed studies of camera images, however, have shown the importance of a different mechanism. What happens is the repeated conjunctions discussed above are prevented by the oscillation. This prevention is due to increasing the distances between each pair of predecessor and follower microbubbles. The distance is made so large that the conjunction ceases to be possible.

\section{T-Z and D-Z Oscillators}

The device used for study of this phenomenon was initially a fluidic oscillator available from earlier experiments described in [11-15]. It made possible elucidating by high-speed camera images the mechanism of the empirically discovered conjunction suppression. Non-optimised initial investigations used a no-moving-part fluidic oscillator (from another project) with dominant geometric parameter, main nozzle exit width $2 \mathrm{~mm}$. The progress made with it was satisfactory and justified making another, dedicated oscillator model as well as applying for the patent document [2], filed in 2006 with colleague Prof. Zimmerman. The earliest publications about the conjunction suppressing phenomenon originated from the University of Sheffield, where the present author tested the design during his temporary stay. It was also there where the oscillator design known as Tesař-Zimmerman or T-Z oscillator was made.

This oscillator, intended for the extensive feasibility tests, consisted of two parts:

(1) An acrylic body with air flow cavity of a fluidic jet-deflection amplifier as shown in Figure 3;

(2) A length of Tygon tube which provided the required delayed-flow negative feedback loop. 


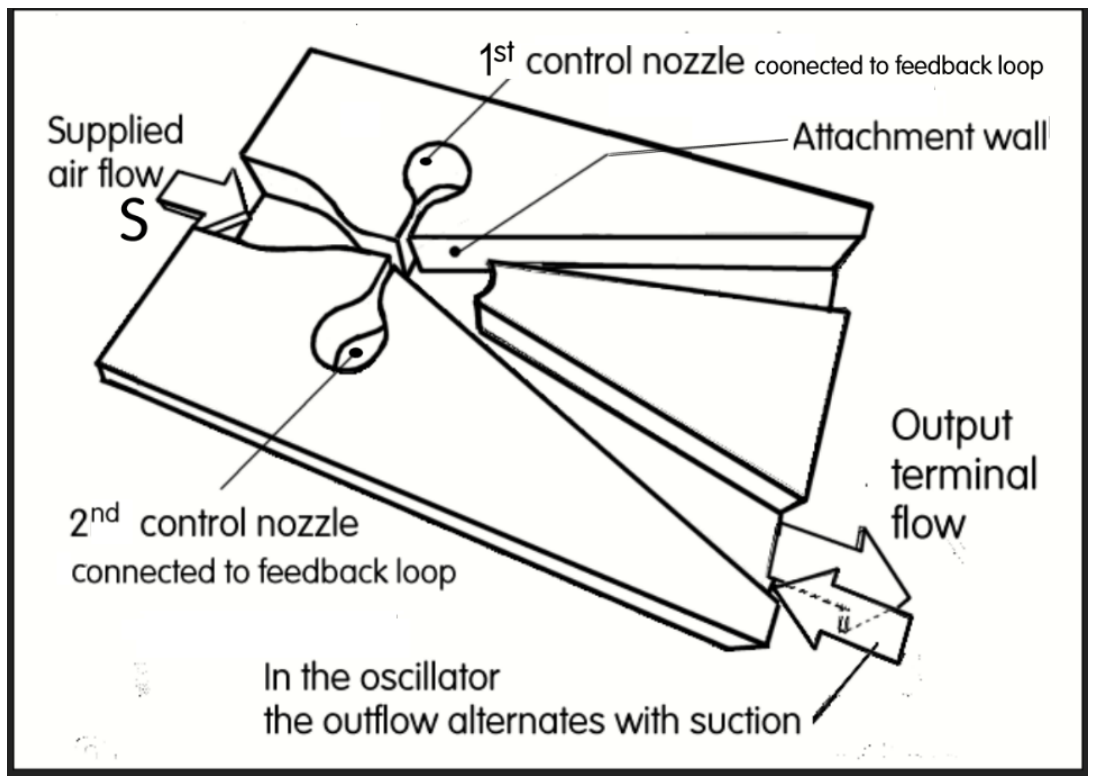

Figure 3. The amplifier used in the Tesař-Zimmerman (T-Z) fluidic oscillator. The jet issuing from the supply $S$ is switched by control flow pulses delivered to either $X_{1}$ or $X_{2}$ and is led by attachment wall to one of the two outputs $Y_{1}$ or $Y_{2}$. Between the individual flow pulses directed out from the output terminal there is suction flow generated by the jet-pumping effect as mentioned in [16].

The amplifier body part, presented in Figure 3, was in this case a stack of four rectangular polymethylmethacrylate (PMMA) plates, with the cavity for air flow made by numerically controlled laser cutting. The most important among these plates is the pair of identical central amplifier plates, each $2 \mathrm{~mm}$ thick. In them were made cavities laser-cut through the whole plate thickness and then the stacked (the reason behind the pair instead of a single thicker plate is a higher precision of generated shapes in thinner plates by the laser-cutting). Both from the bottom and top, the amplifier cavities were then closed by attached cover plates (Figure 3 shows the top plate removed to show the cavities). Useful for identifying the internal flow processes in the amplifier and the role of its components is the schematic representation shown in Figure 4.

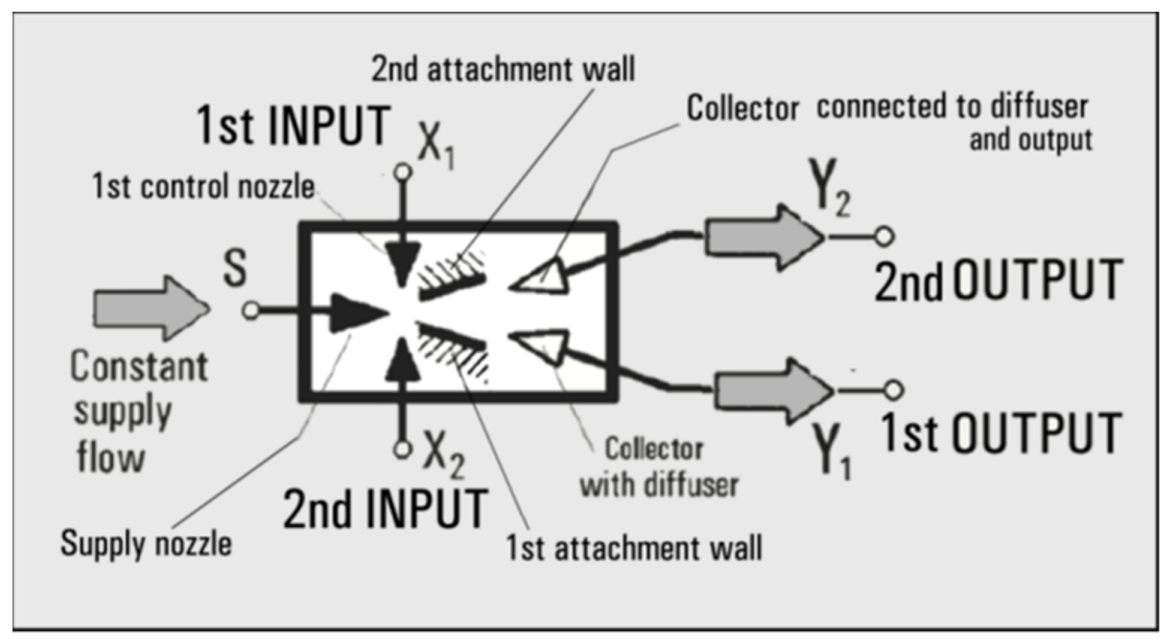

Figure 4. Schematic representation of the amplifier and its components used for drawing circuit diagrams. Black triangles are symbols for nozzles (cross-section area decreases in the flow direction), white triangles are collectors and connected diffusers (cross-section area increasing in the direction of flow). 
Although differing in overall geometry as well as in most details, the fundamental idea on which the oscillation generation is based in [2] was the mutual connection of the input terminals $X_{1}$ and $X_{2}$ by the feedback fluid flow channel presented in Figure 5. This oscillator idea is not new [17]. It is actually already more than 60 years old, as shown in Figure 5 by the copy of original drawing made for patent application. Nevertheless, this earliest version [17] has never been used in any larger scale application. There were only uses in laboratory tests. In 1964, four years later after the patent document from Figure 5, a laboratory model of a similar oscillator was made (for an unidentified military purpose) by a researcher named C. E. Spyropoulos, who was then employed at the Ordnance Fuse Laboratories of Army Materiel Command in the USA. Details of his oscillator are not known, perhaps due to military secrecy. In some literature it is possible to find Spyropoulos mistakenly mentioned as the inventor of this fluidic single-loop oscillator principle.

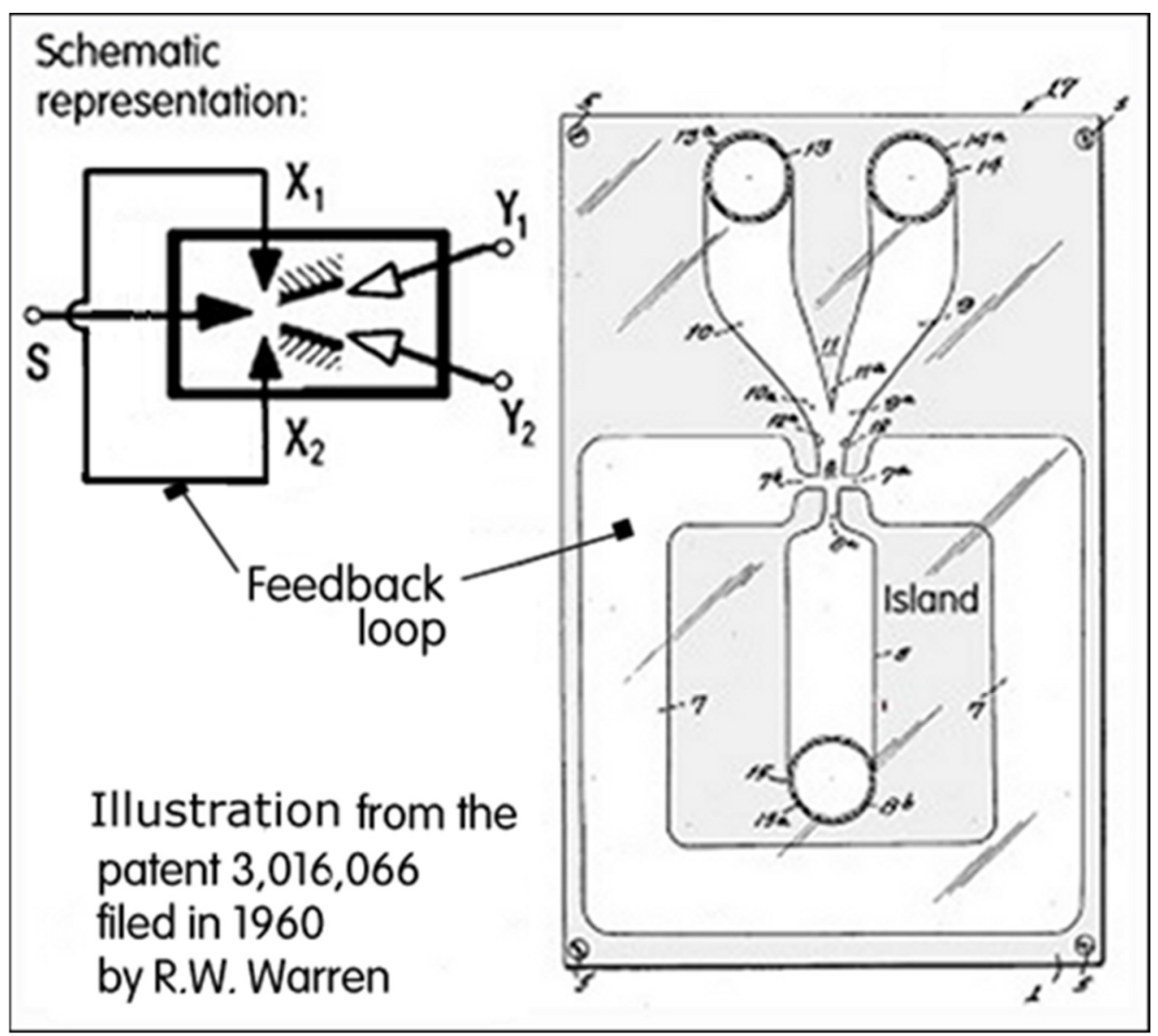

Figure 5. Warren's feedback loop idea [17] of connecting the control terminals is known for over 60 years (the drawing at right is a part of an illustration originated in (1960) but so far it is rarely, if at all, used in an engineering application. The T-Z oscillator that is successfully for generation of microbubbles was developed using this idea.

The microbubble generation could be made also with a different fluidic oscillator design than the one in [2]. Experience [18,19]. has shown, however, that this choice is almost perfect from many points of view, including the economy of oscillator manufacturing.

Figure 4 shows a schematic representation of the amplifier. In this drawing, the air flow comes from the supply terminal $S$ at the left-hand side into the main nozzle and leaves on the opposite right-hand side through one of the two output terminals $Y_{1}$ and $Y_{2}$ in periodic alternation. Inside the amplifier body, the flow from the supply nozzle forms a jet entering the device's interaction cavity. This cavity is a constant-depth space gradually increasing in the flow direction in width and hence also increasing in a cross-sectional 
area. In the symbolic representations, like the one in Figure 4, nozzles are represented by the black triangles gradually decreasing in width in the flow direction, which accelerates the flow. The jet issuing from the supply nozzle is quite sensitive to influences acting in the region where the air flow leaves the supply nozzle. This sensitivity is here used for the amplification effect. At this sensitive location are directed exits from two control nozzles oriented perpendicularly to the main jet flow and connected to control terminals $X_{1}$ and $X_{2}$. Immediately downstream from these two nozzles are two mutually inclined attachment walls. The jet from the main nozzle attaches to one of these walls by the aerodynamic phenomenon called the Coandă effect. In steady flow regimes, it keeps the jet stably attached. Further downstream from the two attachment walls are in Figure 4 two collectors, one of them capturing the jet. Immediately from there, continuing from the collectors towards the exit terminals, are important components, the diffusers. In them the cross-sectional areas for air flow gradually increase. While the schematic symbol for the nozzle is a black triangle, as mentioned above, indicating the area decrease in flow direction and hence acceleration of the flow, the symbol for diffuser is a white triangle. It shows that in the direction of the flow, the flow velocity decreases. The angle between the attachment walls has to be so large that the flow cannot be attached simultaneously to both. Thus only one of the collectors captures the air flow and directs it through the diffuser to the appropriate output terminal. The air pressure in the rectangular region surrounding the amplifier cavities in the centre of Figure 4 is low, usually lower than atmospheric. It is the result of the flow velocity increase in the nozzles at the cost of pressure. As the kinetic energy of the air flowing in the nozzle increases, the pressure inside the region must decrease. This means a low pressure inside the amplifier until the pressure recovery in the diffusers. In the inactive output terminal, this low pressure generates a suction reverse flow. In the oscillator, this means alternating the output flow between positive (in active terminal) to negative (inactive terminal).

The amplifier discussed above is converted into a fluidic oscillator by the addition of the feedback loop channel or tube, connecting the two control nozzles. The earliest found idea in the literature is in patent [17]. The original illustration from this document is shown in Figure 5. It might be said that the aerodynamic design applied there is not really good. Firstly, the gas flow in the feedback loop will experience sudden changes in each of the four sharp corners of the feedback loop. The air flow in them loses its regularity, separates from the wall and generates aerodynamic noise likely to interfere with the carried signal. The second evident disadvantage is the "island" produced by the cutting of the feedback channel. Because of the cutting through the whole plate thickness this part falls out from the PMMA plate during the cutting process. Putting it back and placing it and fixing into the correct position requires another, not easy step in the manufacturing process. A third disadvantage of the drawing in Figure 5 indicates that the designer failed to understand basic problems of internal aerodynamics. He designed the collector and the diffuser connected to it with short and curved walls, with which it is impossible to expect a reasonable pressure recovery. Instead, the diffusers must be long and with a small opening angle.

These shortcomings of Warren's original design [17] were in the T-Z oscillator corrected so that the aerodynamic performance was very much better, as was proven in the performed laboratory experiments. One of the essential improvements made in the T-Z oscillators over the version in Figure 5 is their controllability by varying the feedback loop length, as indicated in Figure 6. This property is particularly of importance with the oscillator output connected to the aerator.

The frequency of generated oscillation is dependent on the feedback tube length, air flow rate, magnitude of the air bleed off through the inactive output terminal, liquid column height in the reactor vessel, and finally also, of course, on the aerator as well as amplifier aerodynamic resistances. These parameters had to be all finely tuned if the bubble coalescence was to be eliminated without any large energetic loss. The results, however, were encouraging. With a standard commercially available sintered alumina 
aerator, as they are used in aquaculture, the capability to generate small microbubbles with an average diameter of $7 \mu \mathrm{m}$ was demonstrated [20]. It demands identifying the so called "sweet spot" [20] of the adjustment. Favourable properties of the T-Z oscillators are demonstrated in the list of successful solutions, those obtained in recent research projects listed below. All cases listed there are of a large future economic potential.

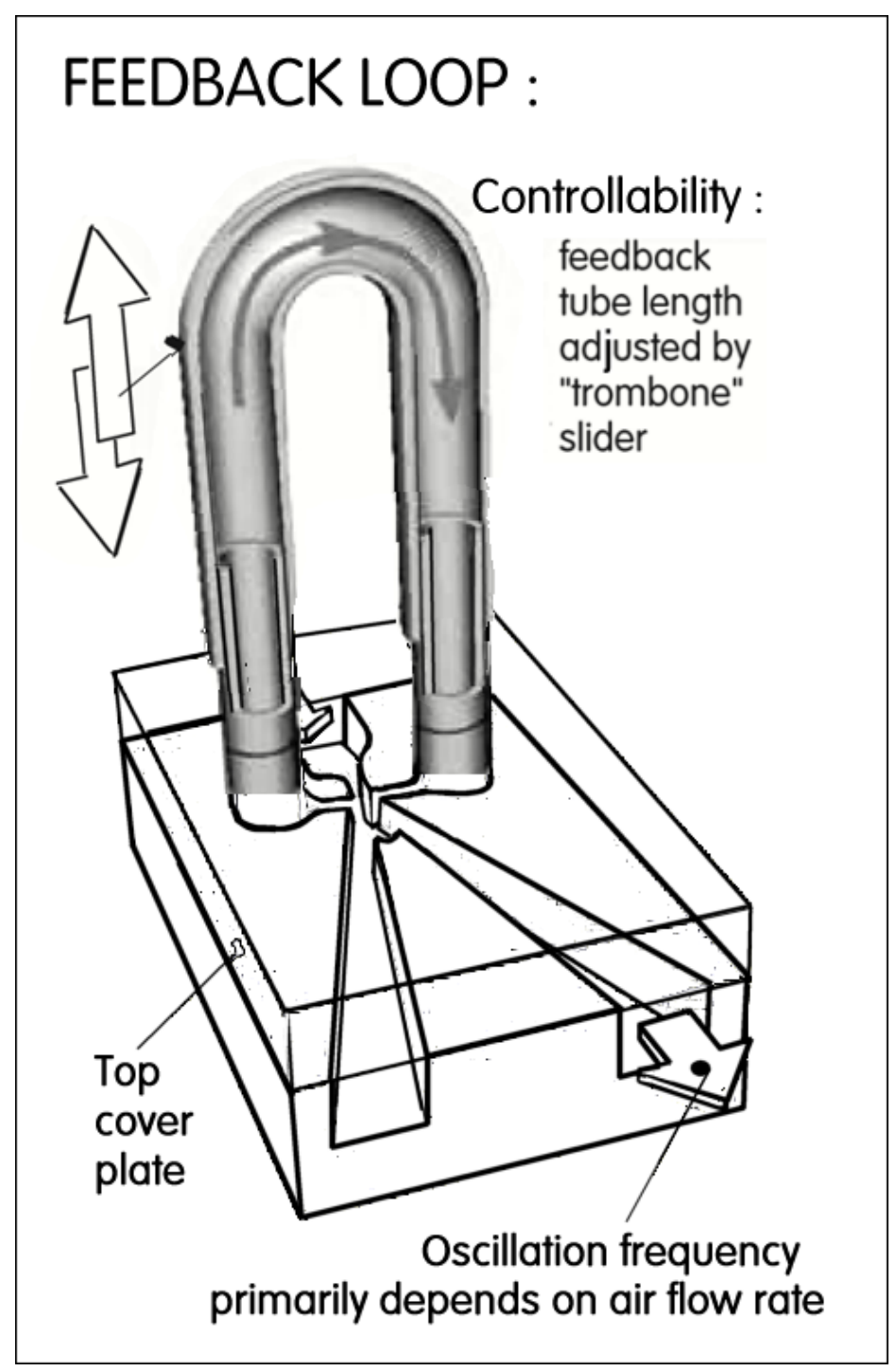

Figure 6. Important property of the T-Z oscillator is its controllability [20]—oscillation frequency adjusting by varying (among other parameters) the feedback loop tube length between the terminals $X_{1}$ and $X_{2}$.

Present development has added to the repertoire of fluidic oscillators in [19] the new jet-deflection design. It is the novel Desai-Zimmerman or D-Z oscillator developed recently at the University of Sheffield, U.K. [21]. Its design was specifically adapted to the conditions prevailing microbubble generating aerators-Figure 7 . The new oscillator version is of two-sided relaxation type, in principle following the one-sided oscillator design of Zalmanzon [22], the earliest known fluidic oscillator but very little is known. The feedback effect is obtained by filling compressed air into its two accumulation chambers $C_{1}, C_{2}$ in this particular design is connected to the middle of the attachment walls. 


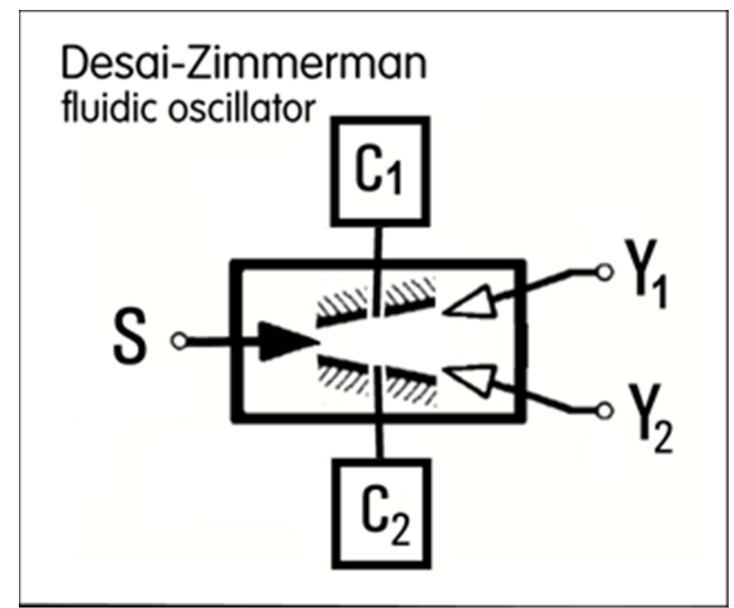

Figure 7. Schematic representation of the new D-Z oscillator developed at the University of Sheffield, U.K. [23] specially for co-operation with microbubble generating aerators. Of the two-sided relaxation type [24], its two accumulation chambers $C_{1}, C_{2}$ connected to the middle of attachment walls are filled by pressure air in alternating manner.

\section{Microbubbles Generated by Mediation of the Oscillator}

Initial targets, at which aimed the microbubble generation mediated by the fluidic oscillators were the obvious advantages obtained from the increase of total mass transport from gas to liquid (or vice versa in gas stripping). This was the result of the increase in the total surface boundary. The sum of mass transport surfaces increases, of course, with increasing the number of bubbles (and hence decreasing their mean diameter) generated from the same gas volume. From the fundamental patent [2], the basis of further developments, is shown in Figure 8 a picture of a typical configuration used in the tests [21,22] with a simple bioreactor (at right-hand side of the picture) and the oscillator (in picture centre). For the photosynthetic growing of primitive organisms [25-29], the reactor walls are transparent and the water inside illuminated by spectrally suitable light. The aerator body is positioned in the centre of the vessel bottom and the bubble motion thus generates a paraxial rising water column. Downwards moving annular water flow is near the reactor walls. This circulation brings the microorganisms periodically to the external illumination.

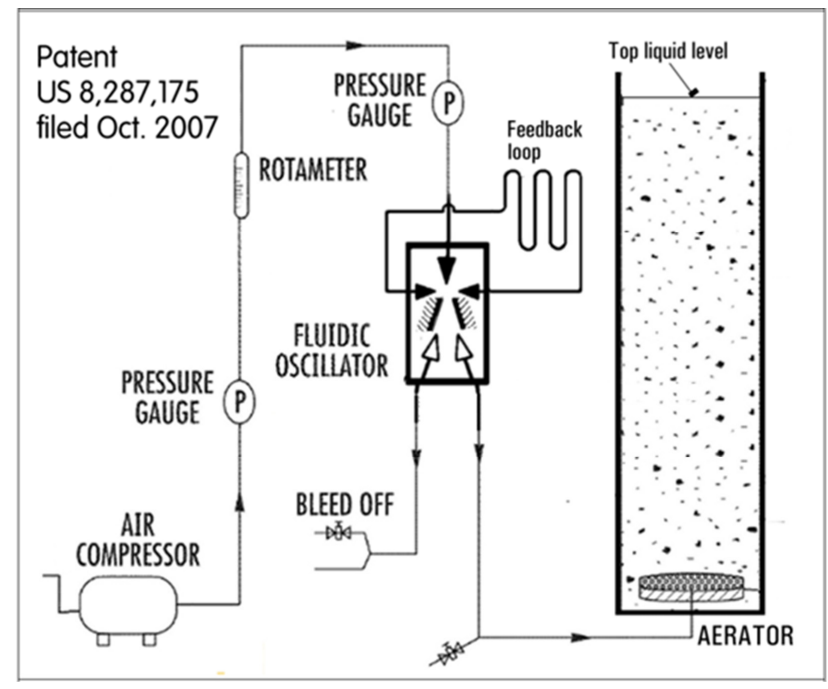

Figure 8. Adapted drawing from the patent document [2] shows the oscillator applied to agitating air flow into a simple bioreactor, a liquid-filled vertical cylinder with transparent walls and the aerator at its bottom. The air bleed off, an obvious energetic loss, was initially necessary for pressure matching. 
In this particular early case, the advantages resultant from the small size of the microbubbles were just used. Their small dimensions correspond to the very small rising velocities according to Equation (1). There was no physically different behaviour from what is known about larger bubbles, just the increased geometric extrapolation to the smaller size. Even then, the enhanced interface area is an obvious benefit for a large number of chemical and biological processes with thus increased heat and mass transfer.

Laboratory experiments have, however, demonstrated various potential improvements resultant from the fact that the properties do not follow a simple linear proportionality law. The patent drawing configuration in Figure 8 presents the setup as it was actually used in laboratory experiment, i.e., with various measurement instrumentations (rotameter for flow rate, pressure gauges) and the bioreactor connected to only one oscillator terminal [2]. The other terminal, in Figure 8, is connected to a bleed-off valve used for "sweet spot" adjustments. It is, of course, not economical to lose in this way a substantial proportion of the compressed air, but this was inevitable for gaining experience with the amplifier loading and matching. Once such an experience was obtained, it became possible to design the T-Z oscillators properly matched to the aerator as the aerodynamic loading. A detailed discussion of (slightly different) fluidic matching problems is available in [30].

The result of the fully matched adjustments, without the lost air flow, is configured with two reactor vessels operated in anti-parallel (suction on one side at the same time as the output flow on the opposite side) as shown in Figure 9.

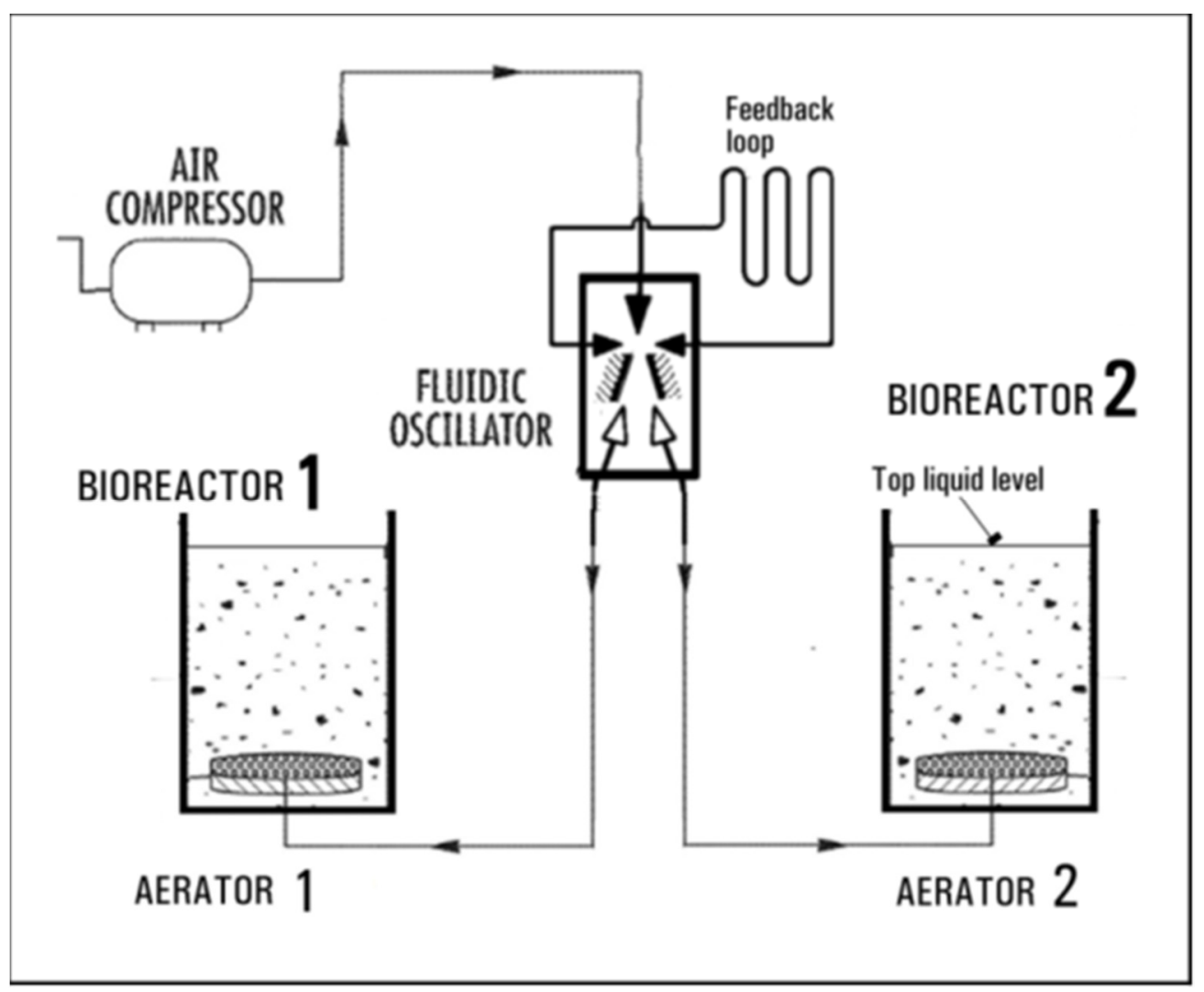

Figure 9. Symmetric two-vessel bioreactor configuration using both output terminals of the jet-deflection oscillator (and its jet-pumping suction flow for each second half cycle). Conditions inside the oscillator and water heights in the vessels require careful matching.

\section{Microbubbles in Flows with the Oscillator}

A matching problem to solve in the generation of microbubbles mediated by the fluidic oscillator depends on bubble properties. It requires knowledge of what the gas 
flow agitating oscillation actually does with the small bubbles. Initially there was a belief that the oscillator simply somehow cuts the larger bubbles into small pieces. The actual process taking place there was discovered using a high-speed camera with extended macro objective lens. It has shown that the effect is actually a suppression of the existence of the conjunctions between several subsequently generated microbubbles. The microbubble trajectories in the vicinity of the pore edge is shown in Figure 10.

The process of the conjunction suppressing begins with the microbubble moving towards the exit inside the aerator pore. The oscillator has to be adjusted so that the microbubble moves near as well as inside the pore back and forth, with the forward portion of the period lasting slightly longer and its intensity slightly more strong. The movement back during each cycle is somewhat weaker, quite naturally, because it is generated by the secondary effect of the jet pumping in the amplifier. The microbubble moves out from the aerator pore (Figure 10) under the action of the oscillator output flow. When the microbubble leaves the pore exit it slows down so that it remains inside the suction radius (cf. Figure 10). Under the subsequent flow back into the pore, the microbubble together with the surrounding liquid also returns and enters the pore. This back flow period is shortlived, however. The return trajectory of the microbubble is thus also short. The oscillator output flow soon thereafter becomes positive (i.e., moving away from the pore) and quite powerfully moves the microbubble away (upwards in Figure 10). In the meantime, another subsequent microbubble comes towards the end of the pore. The essential fact is the two microbubbles now cannot get into the mutual conjunction because the former one is already too far outside of the suction radius.

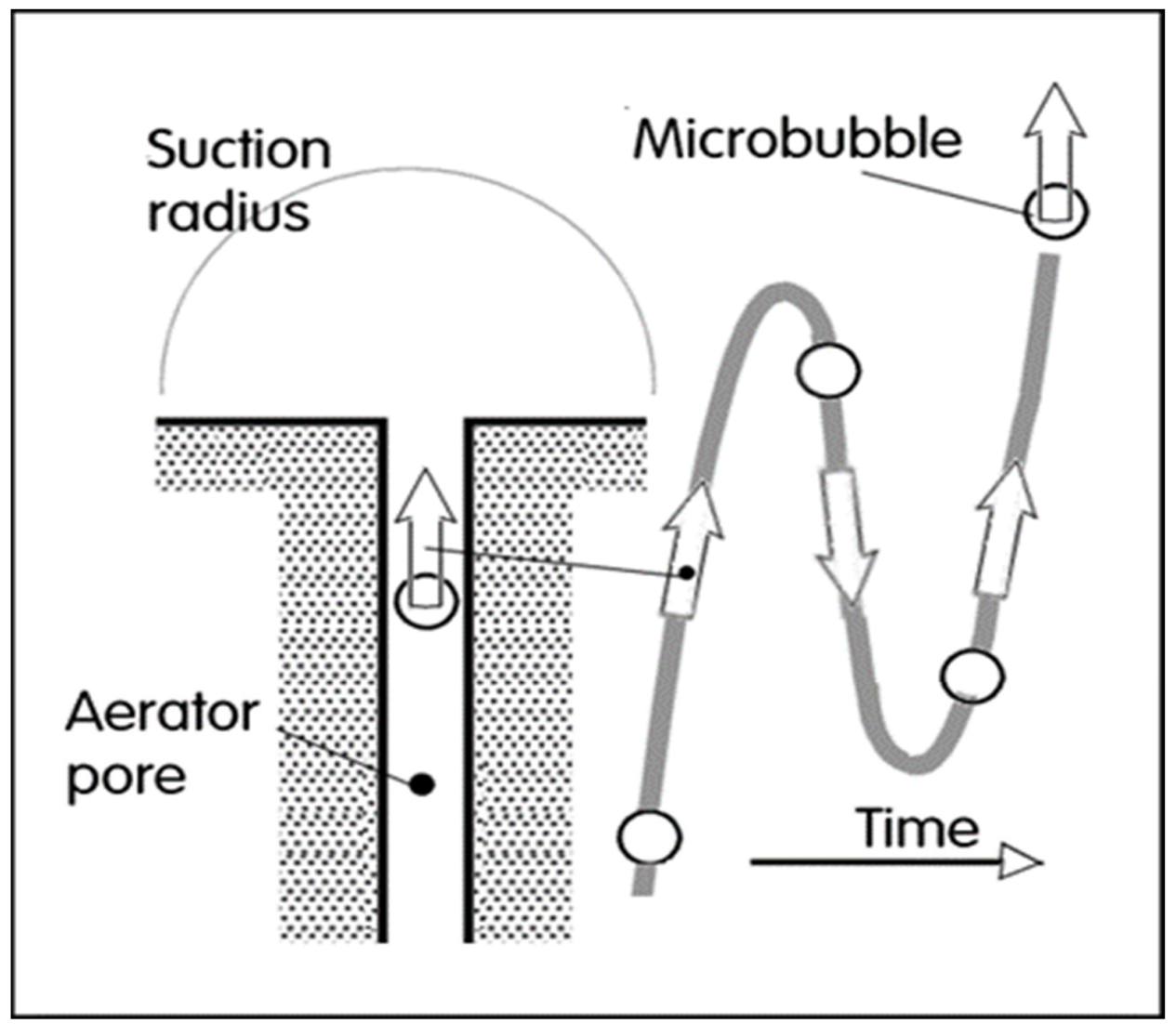

Figure 10. Mechanism of preventing the conjunction in the aerator exit in flow with the oscillator. The microbubbles within the suction radius are in a part of the cycle turned back into the aerator pore-so that they get mutually separated by distance so large that their conjunction is avoided. 


\section{Wastewater Treatment}

Typical use of microbubbles generated by mediation of an oscillator is in the processing of waste water [31]. The present-day municipal wastewaters contain sludge of mostly of organic character. The task of the processing is to decompose the precipitate from sewage into a harmless substance. It is done by aerobic bacteria. Despite various water agitating machineries in the earlier-and even present-treatment plants, typically various paddlewheels, these bacteria in contemporary facilities largely die because of oxygen starvation. This happens in spite of the fact that the largest percentage of the running cost in most processing units today is the mechanical agitation in an attempt to increase the percentage of diluted oxygen, and this cost is increasing. Microbubbles can improve the situation $[25,26]$ by dissolving more oxygen from the atmosphere than earlier methods.

\section{Biotechnology of Micro-Organisms}

An extremely promising application of microbubbles generated with the fluidic oscillator [32] are photobioreactors for growing micro-organisms: algae, bacteria, yeast, and lower fungi. Of particular importance are single-cell microalgae, especially those known as green microalgae, performing photosynthesis. Like the higher green plants they derive the energy for their growth from light-mostly, of course, sunlight. As reactants, they use carbon, $\mathrm{C}$, from $\mathrm{CO}_{2}$ in atmosphere and hydrogen, $\mathrm{H}$, from $\mathrm{H}_{2} \mathrm{O}$. The reaction products are higher hydrocarbons used to build plant body. They are now mostly seen as source of biomass for further processing into biofuels [33] — or even the substrate for food (so far for animal and not human consumption, which may come later). Of particular promise is the photosynthetic generation of lipids, with the advantage of the high speed of biomass growth. Under optimum conditions, the microalgae can double their mass within a few days. An important fact is this is a promising way to decrease carbon in the emissions into the atmosphere and reduce dependence on fossil fuels. In [27,28] was demonstrated their potential to decrease $\mathrm{CO}_{2}$ in the atmosphere-and use it as carbon source together with the produced biomass as feedstock for chemical industry.

At present the largest percentage of investigated microalgae biomass growing is done in open raceways, usually shallow channels aerated by a paddlewheel which simultaneously forces the water to circulate. The aeration effect is poor and yet consumes much energy. More expensive, but with a better future perspective, are closed vessel photobioreactors. They make possible a close control of the growth process and it is in this way that they can demonstrate the microbubble aerator with mediating advantages of the fluidic oscillator $[29,34]$.

While green microorganisms need a supply of $\mathrm{CO}_{2}$ microbubbles for photosynthesis growth, they also need stripping from water inhibiting dissolved $\mathrm{O}_{2}$. This is also a task that can be fulfilled by microbubbles.

Some of the bioreactor application ideas were in principle already mentioned in the literature, but were at that time considered impractical for several reasons. Apart from the most important factor of high costs-running as well capital cost-, the microbubbles generated by the earlier known methods received a quite high input of energy, which in contact with the liquid surface in the top of the bioreactor, caused lysis of living cells. In the microbubbles generated in mediation by fluidic oscillators, this cause of lysis has been practically eliminated, as was found already in the first studies, e.g., [25-29,34,35]. This, together with low cost, simplicity, and absence of driving mechanisms has significantly increased interest in the new generation method. An important factor in the success of the fluidic oscillator in its various roles is its controllability, discussed in [20].

\section{Cleaning, Disinfection and Medical Uses}

Cleaning a contamination by oil emulsion was reported in [36] based on the selective evaporation by the "cold distillation" method based on evaporation dynamics of microbubbles $[37,38]$. Treatment of leachate from landfills based on the same principle is discussed in [39] 
Rather special medical uses of microbubbles were known prior to approach with fluidic oscillators discussed here. The microbubble generation as well as their uses were based on ultrasound. This still constitutes a separate branch of microbubbles, mainly because of focusing their attention on very tiny and hence highly energetic microbubbles, with a typical maximum population at about $1 \mu \mathrm{m}$. At this size the microbubbles are used for increasing ultrasound imaging contrast and also for acoustically controlled locally targeted drug delivery. It remains to be seen whether some of these older applications can also benefit from the new approach with a fluidic oscillator.

Closely related to the practical medical uses is disinfection. There the microbubbles may be larger and this lifted size limit has already shown to be a suitable application for the oscillator mediation in various approaches to disinfection. It is lysis of pathogens to the degree of the remaining medium being unable to cause infective disease. Particularly efficient in this direction may be microbubbles of ozone for ozonisation. In a recent study, this ozonisation was successfully combined with disinfection by non-thermal plasma [40].

\section{Some Recent Results}

An excellent detailed survey of microbubble intensification of bioprocessing by microbubbles was recently published in [20]. What seems to be a different physical process, but in fact is only a consequence of widely different time scales of competing parallel processes, is the evaporation dynamics of microbubbles [37,38]. It results in the absence of the so far unavoidable heating of the liquid, thus making most efficient the "cold distillation". It is likely to become economically of extreme importance, considering the huge quantities of worldwide alcohol production, which thus may be made at a significantly lower cost.

In [41] are presented laboratory tests results of biodiesel production with the microbubble removal of water, as well as esterification, which is important for biodiesel production with a phantom catalyst. There is already a monograph [33] published on the problems associated with biofuels from algae. Several current projects with successful solutions of important tasks are listed in Figure 11.

\section{RECENT SUCCESS CASES}

* Wastewater processing [39]

* Mixing intensification [41]

* Oil separation from emulsion [36]

* Ammonia separation from leachate in minicipal solid waste landfills [40]

* Separation of $\mathrm{CO}_{2}$ from atmosphere

(still in evaluation phase)

Figure 11. Using the T-Z oscillator has already made possible several successful solutions of important tasks, mainly in the field of biotechnology. The supplied air flow agitation by fluidic oscillator was the key factor in its success [25-29]. 
Microbubbles made with oscillator mediation of anaerobic digestion were reported by the authors of [31], demonstrating an increase by $25 \%-100 \%$ in the production rate of biogas.

Interesting results were obtained by comparison in [31] of aeration by $3 \mathrm{~mm}$ diameter bubbles with and without the upstream fluidic oscillation according [42]. The authors' measurements without optimisation have shown at least a 50\% increase in the coefficient of mass transfer. A later optimised study has shown an even substantially higher improvement-a 90\% increase obtained with oscillation. The project [31] was oriented towards the wastewater treatment.

Remarkable increase by as much as $100 \%$ of biogas production was reported with the microbubble mediation of anaerobic digestion of biomass.

Microbubbles were already used in preparation of nanoparticles [43], indicating another association with another rapidly developing area.

\section{Opportunities for the Future}

From various corners came recently a demand for reducing in the atmosphere the demonstrably growing contents of carbon oxides, in particular $\mathrm{CO}_{2}$ [26]. This gas may be separated from the atmospheric air, separately captured, and handled.

Using at a very large scale removed $\mathrm{CO}_{2}$ microbubbles, it may be sequestrated and stored in ocean water (Figure 12) in conditions which offer a better solution than the so far considered underground geology formations. The use of microbubbles is also then available in the catalytic conversion of $\mathrm{CO}_{2}$ and $\mathrm{H}_{2}$ into a combustible product which, because they circulate, may use the existing combustion facilities that are already tried and proven rather than inventing new ones.

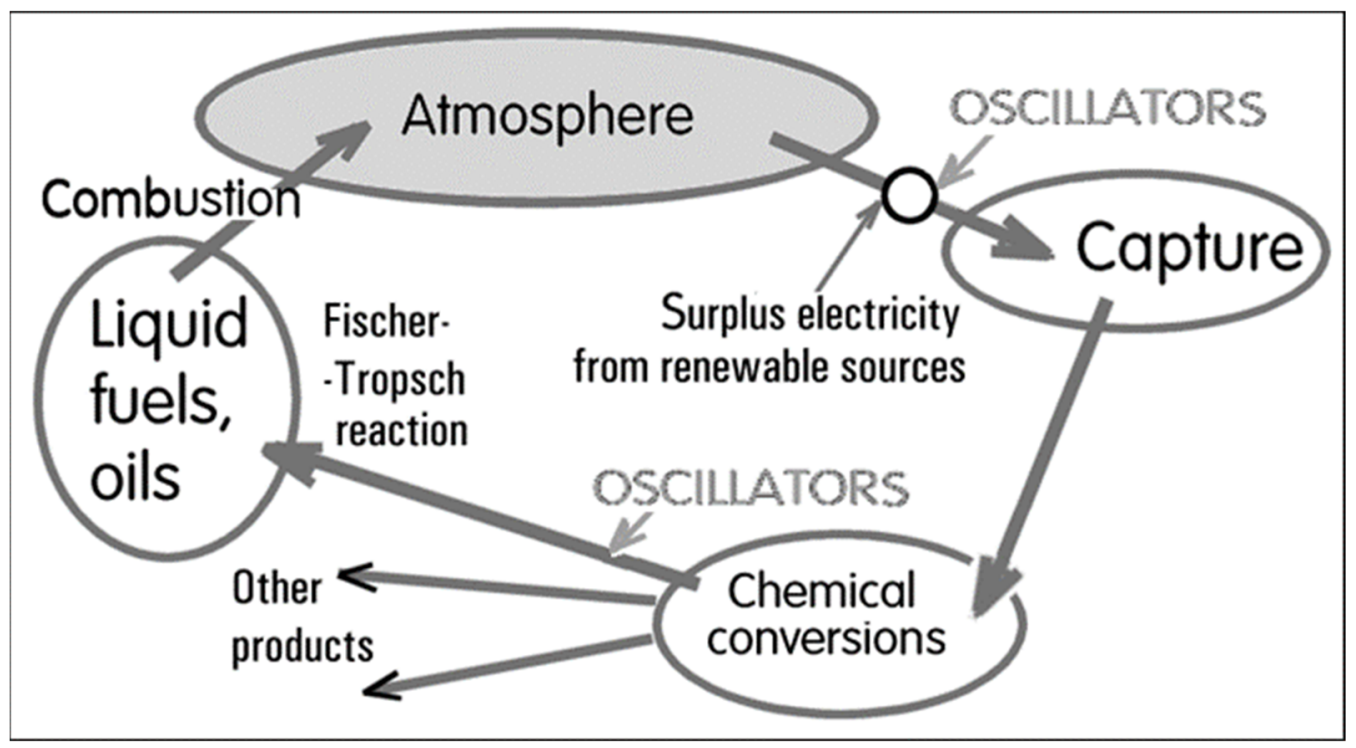

Figure 12. Potential closed-cycle solution of the problem of carbon in the atmosphere: Microbubbles mediated by fluidic oscillators can provide $\mathrm{CO}_{2}$ capture as well as its use in reactions generating combustible products.

\section{Conclusions}

Fluidic oscillators having no moving parts were invented in various alternative versions already in the years 1960-1965. In the meantime since then they have become almost forgotten due to the absence of practical uses in applications. One important use was, however, introduced a decade ago that pushed the oscillators again into the foreground of interest. It is the effect with many uses: Generation of small gas bubbles of a diameter less than $1 \mathrm{~mm}$ by percolation through a large number of parallel pores in an aerator body. There used to be a problem of size discrepancy: Bubbles tended to be larger than 
the pore openings. Microbubbles were initially made small, at a size corresponding to the effective diameter of the aerator pore, but they grew large while still dwelling at the pore exit, where they formed a contact with the next follower bubble, and then with the yet another one and so on. Finally, when it leaves the pore exit, the bubble has become quite large. The fluidic oscillator positioned in series with the aerator solves the problem and manages to keep the microbubbles small. It is done by suppressing the conjunction. The idea of advantages obtained with the microbubbles is not new [44]. They have been known to offer a number of interesting uses, some of them of non-negligible economic importance. However, producing the microbubbles in an energetically efficient way, has been practically impossible.

With a fluidic oscillator agitating periodically the air flow, the microbubbles at the pore exit are kept mutually do distant that their conjunction is out of the question. For this purpose a dedicated fluidic oscillator, the single-loop jet-deflection version, called T-Z was developed. Its feedback loop connects mutually the two control terminals, following essentially the idea of Warren [17]. Compared with the original geometry in Figure 5, this T$\mathrm{Z}$ oscillator has shown improved hydraulic properties as well as the tunability. The paper presents briefly a number of successful application solutions obtained with this oscillator version. Of particular future potential importance may be the carbon cycle presented in Figure 12.

Funding: Author's stay at the Academy was funded by the Ministry of Education, Youth, and Sports of the Czech Republic.

Acknowledgments: Author acknowledges the support by OP RDE grant "Research centre for low carbon energy technologies" number CZ.02.1.01/0.0/0.016_019/0000753 received from the Ministry of Education, Youth, and Sports of the Czech Republic Furthermore, the author gratefully acknowledges for the received institutional support RVO:61388998.

Conflicts of Interest: The authors declare no conflict of interest.

\section{References}

1. Shanavas, J. Surfactants, chemictry, toxixity and remediation. In Pollutant Diseases, Remediation and Recycling; Springer: Berlin, Germany, 2013; Chapter 4.

2. Zimmerman, W.B.; Tesař, V. Bubble Generation for Aeration and Other Purpose. US Patent US 20130092626, 13 August 2019.

3. Tesař, V. Microbubble smallness limited by conjunctions. Chem. Eng. J. 2013, 231, 526-536. [CrossRef]

4. Liger-Belair, G.; Marchal, R.; Robillard, B.; Dambrouck, T.; Maujean, A.; Vignes-Adler, M.; Jeandet, P. On the velocity of expanding spherical gas bubbles rising in line in supersaturated hydroalcoholic solutions: Application to bubble trains in carbonated beverages. Langmuir 2000, 16, 1889. [CrossRef]

5. Okazaki, S. The velocity of ascending air bubbles in aqueous solutions of a surface active substance and the life of the bubble on the same solution. Bull. Chem. Soc. Jpn. 1964, 37, 144. [CrossRef]

6. Haberman, W.L.; Morton, R.K. An Experimental Investigation of the Drag and Shape of Air Bubbles Rising in Various Liquids; Report. 802; David, W., Ed.; Taylor Model Basin: Bethesda, MD, USA, 1953.

7. Okawa, T.; Tanaka, T.; Kataoka, I.; Mori, M. Temperature effect on single bubble rise characteristics in stagnant distilled water. J. Fluid Mech. 1964, 18, 466. [CrossRef]

8. Talaia, M.A.R. Terminal velocity of a bubble rise in a liquid column. Int. J. Math. Comput. Phys. Quantum Eng. 2007, 1, 220.

9. Tesař, V. What can be done with microbubbles generated by a fluidic oscillator? Eur. Phys. J. 2017, 143, 02129. [CrossRef]

10. Tesař, V. Shape oscillation of microbubbles. Chem. Eng. J. 2014, 235, 368. [CrossRef]

11. Tesař, V.; Zhong, S.; Rasheed, F. New fluidic-oscillator concept for flow-separation control. AIAA J. 2013, 51, 397. [CrossRef]

12. Tesař, V.; Trávníček, Z.; Kordík, J.; Randa, Z. Experimental investigation of a fluidic actuator generating hybrid-synthetic jets. Sens. Actuators A 2007, 138, 213-220. [CrossRef]

13. Tesař, V.; Bandalusena, H.C.H. Bistable diverter valve in microfluidics. Exp. Fluids 2011, 50, 1225. [CrossRef]

14. Tesař, V.; Hung, C.-H.; Zimmerman, W.B. No moving part hybrid synthetic jet actuator. Sens. Actuators A 2006, 125, 159. [CrossRef]

15. Tesař, V. Mechanism of pressure recovery in jet-type actuators. Sens. Actuators A 2009, 152, 182. [CrossRef]

16. Tesař, V. Sampling by fluidics and microfluidics. Acta Polytechnica. 2002, 42, 41.

17. Warren, R.W. Fluid oscillator. U.S. Patent 3,016,066, 9 January 1962.

18. Zimmerman, W.B.; Tesař, V.; Bandulasena, H.C.H. Towards energy efficient nanobubble generation with fluidic oscillation. Curr. Opin. Colloid Interface Sci. 2011, 16, 350. [CrossRef]

19. Tesař, V. Taxonomic trees of fluidic oscillators. EPJ Web Conf. 2017, 143, 02128. [CrossRef] 
20. Gilmour, D.J.; Zimmerman, W.B. Microbubble intensification of bioprocessing. In Advances in Microbial Physiology; Elsevier: Amsterdam, The Netherlands, 2020.

21. Al-Mashhadani, M.K.H.; Wilkinson, S.J.; Zimmerman, W.B. Airlift bioreactor for biological applications with microbubble mediated transport processes. Chem. Eng. Sci. 2015, 137, 243. [CrossRef]

22. Desai, P.D.; Ng, W.C.; Hines, M.J.; Riaz, Y.; Tesař, V.; Zimmerman, W.B. Comparison of bubble size distributions inferred from acoustic, optical visualisation, and laser diffraction. Colloids Interfaces 2019, 3, 65. [CrossRef]

23. Desai, P.D.; Zimmerman, W.B. Fluidic Oscillators. Patent GB 1905126, 2020.

24. Abramovic, Z.L. Method of Automatically Controlling Pneumatic or Hydraulic Elements of Instruments and Other Devices. U.S. Patent 3,295,543, 3 January 1967.

25. Hanotu, J.O.; Bandulasena, H.C.H.; Zimmerman, W.B. Microflotation performance for algal separation. Biotechnol. Bioeng. 2012, 109, 1663. [CrossRef]

26. Kasim, M.A.; Meng, T.K. Carbon dioxide $\left(\mathrm{CO}_{2}\right)$ biofixation by microalgae and its potential for biorefinery and biofuel production. Sci. Total Environ. 2017, 584, 1121. [CrossRef]

27. De Morais, M.G.; de Morais, E.G.; Duarte, J.H.; Deamici, K.M.; Mitchell, B.G.; Costa, J.A.V. Biological CO $\mathrm{CO}_{2}$ mitigation by microalgae: Technological trends, future prospects and challenges. World J. Microbiol. Biotechnol. 2019, 13, 78. [CrossRef]

28. Al-Mashhadani, M.K.H.; Wilkinson, S.J.; Zimmerman, W.B. Carbon dioxide rich microbubble acceleration of biogas production in anaerobic digestion. Chem. Eng. Sci. 2016, 156, 24. [CrossRef]

29. Zimmerman, W.B.; Zandi, M.; Bandulasena, H.C.H.; Tesař, V.; Gilmour, D.J.; Ying, K. Design of an airlift loop bioreactor and pilot scales studies with fluidic oscilator-induced microbubbles for growth of a microalga Dunaliella salina. Appl. Energy 2011, 88, 3357. [CrossRef]

30. Tesař, V. Fluidic control of reactor flow_-Pressure drop matching. Chem. Eng. Res. Des. 2009, 87, 817-832. [CrossRef]

31. Rehman, F.; Medley, G.J.D.; Bandulasena, H.; Zimmerman, W.B. Fluidic oscillator-mediated microbubble generation to provide cost effective mass transfer and mixing efficiency to the wastewater treatment plants. Environ. Res. 2015, 137, 32-39. [CrossRef] [PubMed]

32. Zimmerman, W.B.; Hewakandamby, B.N.; Tesař, V.; Bandulasena, H.C.H.; Omotowa, O.A. On the design and simulation of an airlift loop bioreactor with microbubble generation by fluidic oscillation. Food Bioprod. Process. 2009, 87, 215. [CrossRef]

33. Pandey, A.; Lee, D.-J.; Christi, Y.; Soccol, C.R. Biofuels from Algae; Elsevier: Amsterdam, The Netherlands, 2014.

34. Hanotu, J.O.; Zimmerman, W.; Bandulasena, H.; Krunakaran, E.; Biggs, C. Harvesting and dewatering yeast by microflotation. Biochem. Eng. J. 2014, 82, 174. [CrossRef]

35. Hanotu, J.; Kong, D.; Zimmerman, W.B. Intensification of yeast production with microbubbles. Food Bioprod. Process. 2016, 100, 424. [CrossRef]

36. Hanotu, J.; Bandulasena, H.C.H.; Chiu, T.Y.; Zimmerman, W.B. Oil emulsion separation with fluidic oscillator generated microbubbles. Int. J. Multiph. Flow 2013, 56, 119. [CrossRef]

37. Zimmerman, W.B.; Al-Mashhadani, M.K.H.; Bandulasena, H.C.H. Evaporation dynamics of microbubbles. Chem. Eng. Sci. 2013, 101, 865. [CrossRef]

38. Abdulrazzaq, N.N.; Al-Sabbagh, B.H.; Rees, J.M.; Zimmerman, W.B. Purification of bioethanol using microbubbles generated by fluidic oscillation: A dynamical evaporation model. Ind. Eng. Chem. Res. 2016, 250, 1290. [CrossRef]

39. Liu, S.; Wang, Q.H.; Zhan, X.D.; Huang, Q.F.; Huang, P.K. Improved pretreatment (coagulation-floatation and ozonation) of younger landfill leachate by microbubbles. Water Environ. Res. 2010, 82, 657. [CrossRef]

40. Pandhal, J.; Siswanto, A.; Kuvshinov, D.; Zimmerman, W.B.; Lawton, L.; Edwards, C. Cell lysis and detoxification of cyanotoxins using a novel combination of microbubble generation and plasma microreactor technology for ozonation. Front. Microbiol. 2018, 9, 698. [CrossRef]

41. Zimmerman, W.B.; Kokoo, R. Esterification for biodiesel production with a phantom catalyst: Bubble mediated reactive distillation. Appl. Energy 2018, 221, 28. [CrossRef]

42. Zimmerman, W.B.; Tesař, V.; Butler, S.L.; Bandulasena, H.C.H. Microbubble generation. Recent Pat. Eng. 2008, 2, 1. [CrossRef]

43. Du, L.; Wang, Y.J.; Lu, Y.C.; Luo, G.S. Process intensification of BaSO4 nanoparticle preparation with agitation of microbubbles. Powder Technol. 2013, 247, 60. [CrossRef]

44. Desai, P.D.; Hines, M.J.; Riaz, Y.; Zimmerman, W.B. Resonant pulsing frequency effect for much smaller bubble formation with fluidic oscillation. Energies 2018, 11, 2680. [CrossRef] 